\title{
Management of Leukemia and Partial Atrioventricular Septal Defect during Pregnancy
}

\author{
Zhi Li ${ }^{1} \quad$ Haoliang Sun ${ }^{1}$ Jinghang $\mathrm{Li}^{1} \quad \mathrm{Yu} \mathrm{Zhu}^{2}$ \\ 1 jiangsu Province Hospital, the First Affiliated Hospital of Nanjing Medical \\ University, Department of Cardiovascular Surgery, Nanjing, China \\ 2 Jiangsu Province Hospital, the First Affiliated Hospital of Nanjing \\ Medical University, Department of Hematology, Nanjing, China \\ Thorac Cardiovasc Surg Rep 2021;10:e45-e48.
}

\begin{abstract}
Address for correspondence Zhi Li, MD, Jiangsu Province Hospital, the First Affiliated Hospital of Nanjing Medical University, Department of Cardiovascular Surgery, 300 Guangzhou Road, Nanjing 210029, China (e-mail: zhili_cths@163.com).
\end{abstract}

\author{
Abstract \\ Keywords \\ - minimally invasive \\ surgery \\ - congenital heart \\ disease \\ - CHD \\ - hematology \\ - pregnancy
}

\begin{abstract}
Background Pregnancy-associated acute myeloid leukemia (PA-AML) is rare. Cardiac surgery in the context of AML poses challenges that are seldom encountered.

Case Description The subject is a 31-year-old woman at 38 weeks' gestational age diagnosed with AML and partial atrioventricular septal defect. After multidisciplinary consulting, an urgent cesarean section was performed, then chemotherapy was initiated, followed by minimally invasive cardiac surgery with an uneventful recovery. Conclusion Efficient multidisciplinary approach is essential in the management of PAAML and cardiac disease. Minimally invasive cardiac surgery may be safe and useful in patients with AML.
\end{abstract}

\section{Introduction}

Acute myeloid leukemia (AML), generally occurring in older adults, has been reported in 1 out of 75,000 to 100,000 pregnancies. ${ }^{1}$ Current data on clinical features and treatment of pregnancy-associated AML (PA-AML) are scarce. ${ }^{1}$ Complicating $1 \%$ of pregnancies, cardiovascular disease is the leading cause of indirect maternal mortality in developed countries. ${ }^{2}$ Although adult congenital heart disease (CHD) is the most common type of heart disease encountered during pregnancy, partial atrioventricular septal defect (PAVSD) is rare. ${ }^{2}$ Herein, we describe a case with PA-AML and PAVSD who was successfully treated with multidisciplinary treatment including an urgent cesarean section (CS), chemotherapy, and a minimally invasive cardiac surgery.

\section{Case Description}

A 31-year-old Chinese woman at 38 weeks' gestational age was referred to our hospital for progressive dyspnea on exertion and rapid decrease of peripheral blood counts over a 2-month period. The patient had a previous abortion 7 years ago. On examination, she had an elevated respiratory rate of 30 breaths/ min and a pulse oximetry of $91 \%$, and was normotensive $(120 / 88 \mathrm{~mm} \mathrm{Hg}$ ) and tachycardiac (HR $120 \mathrm{bpm})$ with precordial systolic murmur. Her lower extremities were edematous. Her obstetric examination findings and fetal sonography results were normal and appropriately correlated with the gestational age. Peripheral blood counts showed white blood cell $6.22 \times 10^{9} / \mathrm{L}$, hemoglobin $88 \mathrm{~g} / \mathrm{L}$, and platelets $46 \times 10^{9} / \mathrm{L}$. Echocardiography demonstrated a large ostium primum atrial septal defect $(2.1 \mathrm{~cm})$ with significant shunt, cleft left atrioventricular valve with severe insufficiency, patent foramen ovale (PFO) ( - Fig. 1A, B), and moderate tricuspid regurgitation with an ejection fraction of $66 \%$. The pulmonary artery pressure was estimated to be $43 \mathrm{~mm} \mathrm{Hg}$.

After multidisciplinary consulting, an urgent CS was performed under general anesthesia without complications and a morphologic normal female weighting 3,100 g was born. The mother's bone marrow aspirate revealed a diagnosis of AML. Fluorescence in situ hybridization karyotyping was positive for $\mathrm{t}(8: 21)$ (q22;q22). After the CS, the patient's vital signs and received

September 9, 2020

accepted after revision

November 16, 2020
DOI https://doi.org/

10.1055/s-0041-1722876.

ISSN 2194-7635. (c) 2021. The Author(s).

This is an open access article published by Thieme under the terms of the Creative Commons Attribution-NonDerivative-NonCommercial-License, permitting copying and reproduction so long as the original work is given appropriate credit. Contents may not be used for commercial purposes, or adapted, remixed, transformed or built upon. (https://creativecommons.org/ licenses/by-nc-nd/4.0/)

Georg Thieme Verlag KG, Rüdigerstraße 14, 70469 Stuttgart, Germany 


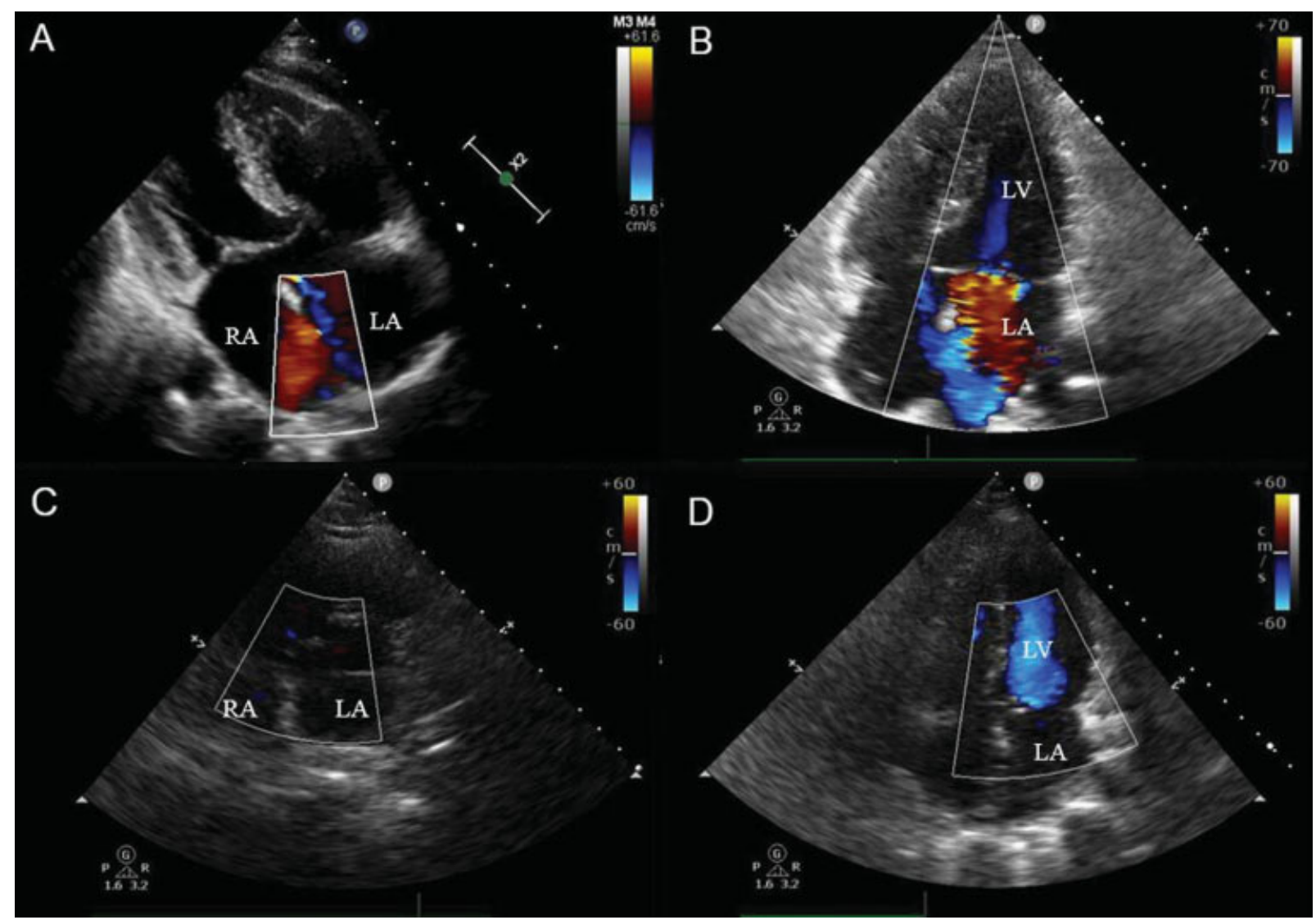

Fig. 1 Preoperative TTE showed (A) an ostium primum ASD and PFO, and (B) severe left-sided atrioventricular valve insufficiency. Postoperative TEE confirmed an intact septum without residual shunt (C) and trace mitral insufficiency (D) after surgery. ASD, atrial septal defect; LA, left atrium; LV, left ventricle; PFO, patent foramen ovale; RA, right atrium; TTE, transesophageal echocardiography.

oxygenation gradually returned to normal values. Then induction chemotherapy was initiated, a complete remission obtained, followed by two cycles of consolidation chemotherapy. Subsequently video-assisted cardiac surgery was performed to correct her cardiac defects. We used a 5-cm right submammary groove approach with a $5-\mathrm{mm}$ endoscope to enhance intracardiac visualization. Peripheral cannulation was used for cardiopulmonary bypass. A vertical right atriotomy incision was used to expose and incise the septum primum, which allowed repair of the left atrioventricular valve. The cleft was closed with a continuous 5/0 Prolene suture, and the ostium primum defect was repaired with the use of an autologous pericardial patch (-Fig. 2). PFO was closed with a horizontal mattress pledgeted 4/0 Prolene suture. A Sorin $28-\mathrm{mm}$ ring was implanted for tricuspid annuloplasty. No blood product was given perioperatively. Postoperative echocardiography demonstrated no residual shunt and trace mitral insufficiency ( - Fig. 1C, D). The patient was extubated in the sixth hour after surgery and discharged home without complication after 9 days in hospital. The patient and the infant are alive and in good health after a follow-up of 12 months.

\section{Discussion}

Signs and symptoms experienced during a normal pregnancy are similar to those of cardiac disease, leading to a confusing clinical picture. ${ }^{3}$ The clinical feature of AML presenting during pregnancy is also similar to that of nonpregnant women. ${ }^{1}$ Thus, the diagnosis can be easily delayed. In our case, the patient lived in a remote area. Her CHD was unnoticed until her visit to our hospital. The diagnosis of PA-AML and PAVSD was confirmed very late (at 38 weeks' gestational age), partly because her symptoms were nonspecific.

Preexisting cardiac conditions can interact with the physiological changes occurring during pregnancy and increase the risk of maternal and fetal adverse outcomes. ${ }^{3}$ Our patient had hepatomegaly and edematous lower extremities, and the echo showed a dilated right ventricle and atrium with elevated pulmonary systolic pressure, which confirms the diagnosis of right heart failure. Acute leukemia may present with hyperleukocytosis, thromboses, or disseminated intravascular coagulation, in the context of a gestational associated thrombogenic milieu. ${ }^{1}$ Hemorrhage secondary to acute leukemia is also common. ${ }^{1,4}$ Thus, the treatment of PA-AML and PAVSD is extremely challenging. Our patient fell into extremis, developing right heart failure. Taking into account the immediate threat to the lives of the woman and fetus, emergent CS was promptly accomplished. For PA-AML, chemotherapy remains the mainstay of treatment. ${ }^{4}$ Based on the available data, PA-AML treated with appropriate regimens might obtain outcomes similar to those of nonpregnant woman, and the prognosis is worse only when appropriate treatment is significantly delayed. ${ }^{4}$ In our case, urgent cardiac surgery was abandoned out of concern for 


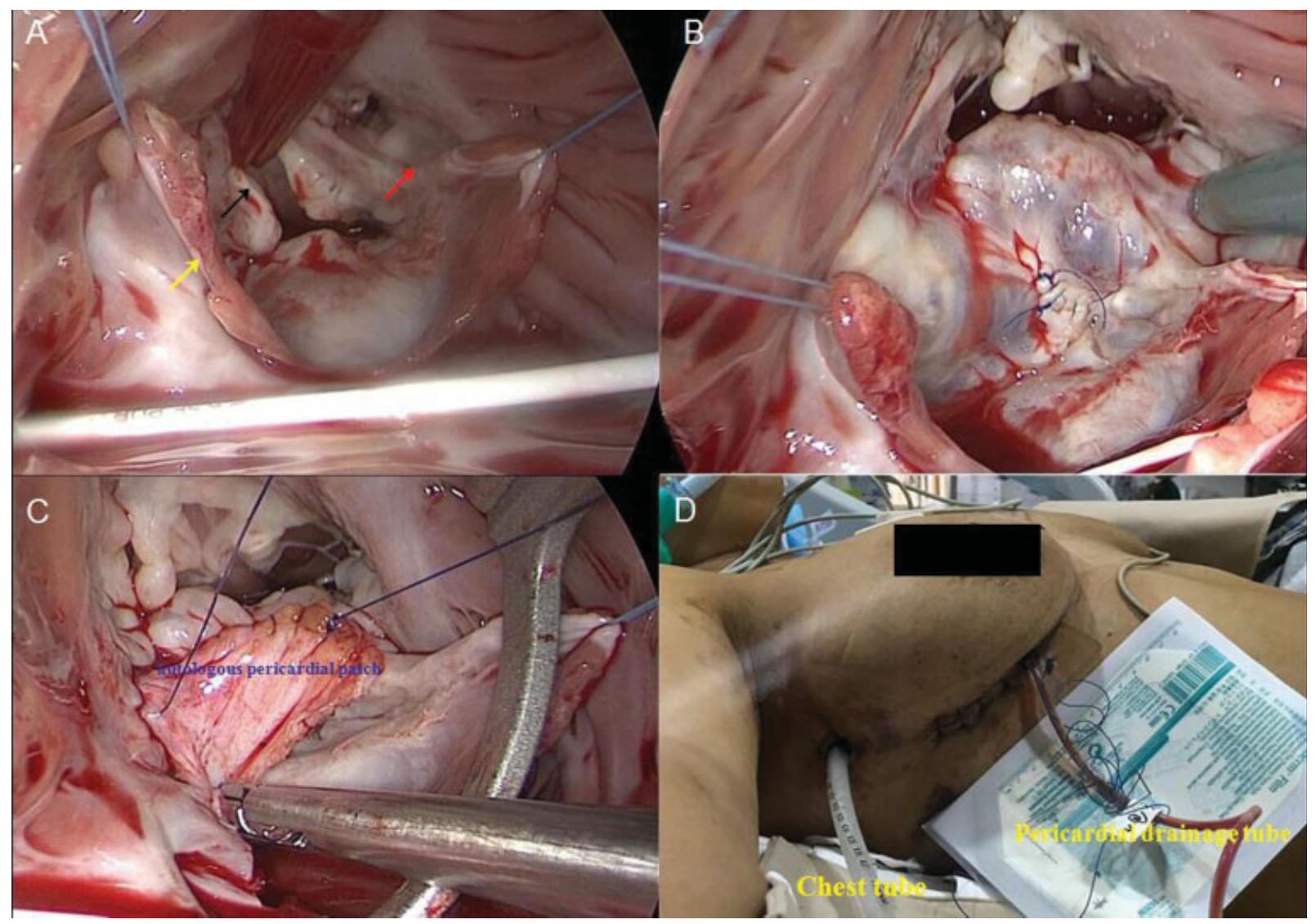

Fig. 2 (A) Cleft between the left superior and inferior leaflets (black arrow) was examined and repaired through the ostium primum ASD (red arrow) and the incised septum primum (yellow arrow). (B) Saline test showed a competent mitral valve after the repair. (C) An autologous pericardial patch was use to repair the ostium primum ASD. (D) Postoperative appearance of the patient's chest. ASD, atrial septal defect.

the potentially fatal uterine bleeding associated with cardiopulmonary bypass and the delay to initiate chemotherapy which would negatively affect the mother's prognosis.

To our knowledge, this is the first case of PAVSD in PA-AML with subsequent minimally invasive cardiac surgery. AML is characterized by uncontrolled proliferation of immature, abnormal blast cells, and impaired production of normal blood cells. ${ }^{1,4}$ Surgical trauma and cardiopulmonary bypass, because of their immune-depressing effects, have the potential risk of increasing the hematological problems, leading to fatal or morbid complications. ${ }^{5}$ The current mortality risk calculators used for cardiac surgery do not account for hematological malignancy, but several studies have concluded that patients with hematological malignancy carry an increased risk of complications. ${ }^{5}$ To reduce the risk of postoperative infection and thrombohemorrhagic complications, we performed the cardiac surgery after obtaining complete remission and we waited until two cycles of consolidation treatment were done to confirm that there was no relapse.

Because of its good exposure, median sternotomy has been the conventional approach for cardiac surgery. However, it causes long-term adverse effects on patients with large trauma, bleeding, long-term hospitalization, and obvious scars after recovery. Recently, minimally invasive surgery has gained popularity as it is associated with decreased blood loss, less postoperative pain, shorter hospitalization time, faster recovery, and better cosmesis. ${ }^{6}$ Minimally invasive cardiac surgery is also related to reduced inflammatory reaction and coagulopathy. ${ }^{7}$ It is thus believed that high-risk patients benefit most from minimally invasive surgery. Our AML patient clearly derived healing benefits from the minimally invasive surgery, avoiding issues with sternal healing and postoperative infections. Totally robotic repair of PAVSD has also been reported, ${ }^{8}$ but the high expenses associated with it cannot be overlooked. The long operation time was thought to be a shortcoming of minimally invasive cardiac surgery. However, the operative duration can be significantly shortened with surgical experience gained. ${ }^{8}$

\section{Conclusion}

In summary, this is a rare case of the very unusual coincidence of PA-AML and PAVD at full-term pregnancy. Efficient multidisciplinary approach is essential in management. Minimally invasive surgery may be safe and useful in patients with AML.

Conflict of Interest

None declared.

\section{References}

1 Horowitz NA, Henig I, Henig O, Benyamini N, Vidal L, Avivi I. Acute myeloid leukemia during pregnancy: a systematic review and meta-analysis. Leuk Lymphoma 2018;59(03):610-616 
e48 Management of Leukemia and Partial Atrioventricular Septal Defect during Pregnancy Li et al.

2 Lindley KJ, Conner SN, Cahill AG. Adult congenital heart disease in pregnancy. Obstet Gynecol Surv 2015;70(06):397-407

3 Martin S, Arafeh J. Cardiac disease in pregnancy. AACN Adv Crit Care 2018;29(03):295-302

4 Ali S, Jones GL, Culligan DJ, et al;British Committee for Standards in Haematology. Guidelines for the diagnosis and management of acute myeloid leukaemia in pregnancy. Br J Haematol 2015;170 (04):487-495

5 Guler A, Sahin MA, Cingoz F, Ozal E, Demirkilic U, Arslan M. Can cardiac surgery be performed safely on patients with haematological malignancies. Cardiovasc J Afr 2012;23(04):194-196
6 Langer NB, Argenziano M. Minimally invasive cardiovascular surgery: incisions and approaches. Methodist DeBakey Cardiovasc J 2016;12(01):4-9

7 Paparella D, Rotunno C, Guida P, et al. Minimally invasive heart valve surgery: influence on coagulation and inflammatory response. Interact Cardiovasc Thorac Surg 2017;25(02): 225-232

8 Gao C, Yang M, Xiao C, Zhang H. Totally robotic repair of atrioventricular septal defect in the adult. J Cardiothorac Surg 2015; 10:156 